\title{
The Association between Platelet count and Liver volume after eradication of Hepatitis $C$ virus by Direct-acting Antivirals: a retrospective study
}

Yuya Seko ( $\nabla$ yuyaseko@koto.kpu-m.ac.jp )

Kyoto Prefectural University of Medicine https://orcid.org/0000-0002-3658-5894

Michihisa Moriguchi

Kyoto Prefectural University of Medicine

Aya Takahashi

Kyoto Prefectural University of Medicine

Shinya Okishio

Kyoto Prefectural University of Medicine

Seita Kataoka

Kyoto Prefectural University of Medicine

Keiichiroh Okuda

Kyoto Prefectural University of Medicine

Naoki Mizuno

Kyoto Prefectural University of Medicine

Masashi Takemura

Kyoto Prefectural University of Medicine

Hiroyoshi Taketani

Kyoto Prefectural University of Medicine

Atsushi Umemura

Kyoto Prefectural University of Medicine

Taichiro Nishikawa

Kyoto Prefectural University of Medicine

Kanji Yamaguchi

Kyoto Prefectural University of Medicine

Yoshito Itoh

Kyoto Prefectural University of Medicine

Research article

Keywords: DAA, SVR, liver volume, platelet count 
Posted Date: December 9th, 2019

DOI: https://doi.org/10.21203/rs.2.18465/v1

License: (c) (i) This work is licensed under a Creative Commons Attribution 4.0 International License. Read Full License 


\section{Abstract}

Background: Although most patients who obtain sustained virological response (SVR) show improved liver function, some patients show decreased platelet counts after eradication of hepatitis $\mathrm{C}$ virus (HCV). The aim of this retrospective study was to clarify the association of liver and spleen volumes with platelet count after SVR achieved by direct-acting antiviral (DAA) treatment.

Methods: This study enrolled 36 consecutive patients treated by DAAs who obtained SVR between September 2014 and December 2018. Liver and spleen volumes were derived from computed tomography scans obtained at pretreatment, SVR, and 48 weeks after SVR. No patient developed hepatocellular carcinoma during this study.

Results: Compared with pretreatment, the median AST, ALT, albumin serum levels, and platelet counts improved significantly at SVR and 48 weeks after SVR. The liver/spleen volumes per body weight were decreased significantly from $22.5 / 4.2 \mathrm{~mL} / \mathrm{kg}$ to $21.1 / 3.6 \mathrm{~mL} / \mathrm{kg}$ at 48 weeks after SVR. The change in liver volume was associated with change in platelet count, and the change in spleen volume was negatively associated with change in serum albumin level. Multivariate analysis identified change in liver volume ( $\geq 95 \%$, odds ratio $76.9, \mathrm{P}=0.005$ ) as the factor associated with improvement in the platelet count at 48 weeks after SVR. The patients with increased liver volume at 48 weeks after SVR showed increased platelet count.

Conclusions: Both liver and spleen volume once decreased significantly by eradication of HCV. The patients with re-increased liver volume showed rapid increase of platelet count.

\section{Introduction}

Hepatitis $\mathrm{C}$ virus ( $\mathrm{HCV}$ ) infection is one of the most important public health concerns in the world. An estimated 270-300 million people are infected worldwide, and the prevalence of the infection is expected to peak in the next 10-20 years [1]. The proportion of patients obtaining sustained virological response (SVR) due to direct-acting antiviral (DAA) treatment subsequently dramatically increased to more than $95 \%$ [2].

Most patients treated by DAAs show improved liver function after obtaining SVR. However, some patients have persistently abnormal liver function test results after SVR. Platelet counts also improved after SVR, and the change in platelet counts was greater in patients obtaining SVR than patients without SVR after IFN-based therapy [3]. Thrombocytopenia has been associated with Hepatocellular carcinoma (HCC) development in cirrhotic patients with HCV infection [4]. On the other hand, several studies showed that thrombocytosis is associated with bigger tumor size, more frequent metastasis and poor prognosis [5-7] Though it is unclear if thrombocytopenia or thrombocytosis per se is a risk factor for HCC development, evaluating the predictive factor of platelet count after SVR is important. 
Liver and spleen volumes derived from computed tomography (CT) scans have been used to assess graft volumes, and to predict operative outcomes in patients with decompensated liver disease [8,9]. Several study reports have discussed the relationship between liver volume and functional reserve in patients with cirrhosis[10-12]. In cirrhotic patients, liver volume decreased in accordance with progression of hepatic fibrosis, and liver and spleen volumes might be related to the degree of portal hypertension[1315]. However, the association between remodeling changes in liver/spleen volume and changes in platelet count in patients undergoing DAA therapy has remained unclarified.

The aims of this study were to assess changes in liver/spleen volumes and platelet counts over a 2-year period in patients who obtained SVR by DAA therapy, and to identify the predictors of increased platelet counts.

\section{Materials And Methods}

\section{Patients}

We performed a retrospective single-center study of consecutive patients with HCV infections from the outpatient clinic at the Department of Gastroenterology and Hepatology, Kyoto Prefectural University of Medicine, from September 2014 to December 2018. All patients had started an oral DAA regimen (dacratasvir [DCV], asunaprevir [ASV], sofosbuvir [SOF], ledipasvir [LDV], ombitasvir [OBV], ritonavirboosted paritaprevir [PTV/r]) for 12 to 24 weeks. An SVR was defined as undetectable HCV RNA at 24 weeks after the end of treatment. The exclusion criteria were any other liver diseases, including autoimmune hepatitis, drug-induced liver disease, and primary biliary cholangitis. Patients with ChildPugh class $B$ and $C$ cirrhosis or who developed hepatocellular carcinoma (HCC) during this study period were excluded because only patients with Child-Pugh A could had DAA treatment in Japan and anticancer therapy such as liver resection or radio frequency ablation effect liver volume. Among 248 patients undergoing DAA therapy, 77 patients were diagnosed Child-Pugh class A liver cirrhosis. We excluded 5 patients who developed HCC in follow-up period and 36 patients who were lack of CT imaging data. After that, 36 patients with Child-Pugh class A who underwent CT imaging at pretreatment, SVR, and 48 weeks after SVR were enrolled in this study. Patients underwent CT imaging every 6-12 months to identify the incidence of HCC. All patients provided information on demographic factors, medical history, and medication usage. Type 2 diabetes mellitus was diagnosed according to the Report of the Expert Committee on the Diagnosis and Classification of Diabetes Mellitus. No patients had been administered growth factors such as IL-11 agent. All patients provided written informed consent about this study at the start of DAA treatment, and this study was conducted in accordance with the 2013 Declaration of Helsinki and approved by institutional ethical committee (ERB-C-588-1).

\section{Laboratory imaging and clinical parameters}

Laboratory assays included blood cell counts and measurements of serum concentrations of aspartate aminotransferase (AST), ALT, gamma glutamyl transpeptidase (GGT), albumin, total cholesterol, 
triglycerides, and alpha-fetoprotein (AFP). Body mass index (BMI) was calculated as weight $(\mathrm{kg}) / \mathrm{height}$ $\left(\mathrm{m}^{2}\right)$. The FIB-4 index was calculated using the following formula: FIB-4 index $=([\mathrm{age}(\mathrm{yr}) \times \mathrm{AST}$ $(\mathrm{U} / \mathrm{L})] /$ platelet count $\left.\left[10^{9} / \mathrm{L}\right]\right) \times(\mathrm{ALT}[\mathrm{U} / \mathrm{L}])^{1 / 2}$. After CT scanning, 1-mm portal vein phase reconstruction images obtained from raw data were divided and transferred to workstations (Ziosoft Inc, Tokyo, Japan). The liver and spleen volume assessments of CT examinations were calculated automatically by workstation software. All parameters were evaluated at pretreatment, SVR, and 48 weeks after SVR. All the CT studies performed in the same type of scanners with using same protocol.

\section{Statistical analysis}

The liver volume per body weight (LV/BW) and spleen volume per body weight (SV/BW) were calculated at each point. The change in biological and imaging parameters at 48 weeks after SVR from those at baseline were shown as delta (\%). Distributions of the characteristics of the study patients were assessed by the chi-squared test or Mann-Whitney $U$ test, as appropriate. Pearson coefficient were performed to analyze the factors associated with change in liver and spleen volume. In multivariate logistic regression analysis, platelet count, albumin, total bilirubin, and change in LV/BW at baseline to 48 weeks after SVR were included as confounders. Odds ratios (ORs) and 95\% confidence intervals (Cls) were also calculated. Statistical comparisons were performed by SPSS software (SPSS Inc., Chicago, IL). All Pvalues less than 0.05 by the two-tailed test were considered significant.

\section{Results}

\section{Patient characteristics}

Table 1 shows the characteristics of the patients at the start of DAA therapy who achieved SVR. This study included 36 patients (23 [63.9\%] males) with a median age of 68 years. The distributions of DAA therapies were as follows: DCV/ASV $n=12(33.3 \%)$, SOF/LDV $n=22(61.1 \%)$, and SOF/ribavirin $n=2$ (5.6\%). At the start of treatment, $22.2 \%$ of patients were alcohol drinkers, $11.1 \%$ had fatty liver, and $25.0 \%$ had varices. Among the 36 patients, $22(61.1 \%)$ patients had a history of HCC. Seven patients were treated with radio frequency ablation, and 15 patients were treated with transcatheter arterial chemoembolization. The median liver volume per body weight (LV/BW) was $22.5 \mathrm{~mL} / \mathrm{kg}$ and median spleen volume per body weight (SV/BW) was $4.2 \mathrm{~mL} / \mathrm{kg}$.

\section{Changes in biochemistry and liver/spleen volumes}

The median serum levels of AST and ALT were significantly lower at SVR and 48 weeks after SVR than the levels at baseline (Figure 1). These values did not change significantly between SVR and 48 weeks later (Figure 1). The median platelet count and serum albumin level were significantly increased at SVR and 48 weeks after SVR than the levels at baseline (Figure 1). These values also did not change significantly between SVR and 48 weeks later (Figure 1). The median values of LV/BW decreased 
significantly from $22.5 \mathrm{~mL} / \mathrm{kg}$ at baseline to $21.1 \mathrm{~mL} / \mathrm{kg}$ at SVR. The median value did not change significantly between SVR and 48 weeks later (Figure 2). However, the SV/BW decreased significantly between baseline $(4.2 \mathrm{~mL} / \mathrm{kg})$ and SVR, between SVR and 48 weeks after SVR $(3.6 \mathrm{~mL} / \mathrm{kg})$ and between baseline and 48 weeks after SVR (Figure 2). The change in LV/BW (\%) correlated with change in platelet count $(P=0.007)$. The change in SV/BW $(\%)$ correlated with the platelet count at baseline $(P=0.032)$ and SV/BW (\%) the negatively correlated with the change in albumin level $(P=0.005)$ (Figure 3$)$. The platelet count, LV/BW, and SV/BW were not different between ribavirin-include and ribavirin-free regimen.

\section{Factors associated with improvements in platelet counts}

The median platelet counts increased significantly from $10.3 \times 10^{4} / \mu \mathrm{L}$ at baseline to $11.6 \times 10^{4} / \mu \mathrm{L}$ at 48 weeks after SVR. Among the study population, 10 (27.8\%) patients showed a platelet count that decreased from baseline to 48 weeks after SVR. Table 2 lists the characteristics of study patients stratified by changes in their platelet counts. The differences between gender, age, BMI, alcohol intake, and the prevalence of complications between the patients whose counts increased and those whose counts decreased were not significant. The baseline liver function tests of the 2 groups also did not reflect the changes in platelet counts. The median LV/BW values at baseline of the patients with decreased platelet count vs those with increased platelet count were $24.0 \mathrm{~mL} / \mathrm{kg}$ and $21.1 \mathrm{~mL} / \mathrm{kg}$, respectively $(P=0.037)$. The difference between median SV/BW values at baseline in the patients with decreased platelet count vs those with increased platelet count was not significant. The change in median LV/BW value of patients with decreased platelet counts between baseline to 48 weeks after SVR $(87 \%)$ was significantly smaller than the change in median value of patients with increased platelet count $(97 \%)(P<0.001)$. Differences between the changes in SV/BW values were not significant.

To identify factors significantly associated with improved platelet counts, we performed a multivariate logistic regression analysis. The following factors were evaluated by multivariate analysis: platelet count, albumin, total bilirubin, change in LV/BW at baseline to 48 weeks after SVR. The change in LV/BW at 48 weeks after SVR ( $\geq 95 \%)$ was identified to be an independent factor associated with improved platelet count (adjusted OR 76.9, 95\% Cl 3.78-1000; $P=0.005$ ) (Table 3).

\section{Association between liver volume and platelet count.}

To evaluate the relationships between liver volume and platelet counts, we followed the changes in LV/BW values according to the changes in platelet counts from baseline to 48 weeks after SVR; in the patients with increased and the patients with decreased platelet counts at 48 weeks after SVR (Figure 4). In patients with increased platelet counts, the LV/BW at SVR and 48 weeks later compared to baseline were 0.98 and 1.02, respectively. In patients with decreased counts at 48 weeks after SVR, the changes were 0.90 and 0.83 , respectively. The changes at each point in LV/BW values of the patients with increased platelet counts were significantly greater than the changes in the values of the patients with decreased platelet counts $(P=0.017, P<0.001$, respectively). We followed the LV/BW value of 24 
patients at 96 weeks after SVR. The rest of 12 patients were excluded due to lack of data or development of HCC (Figure 4). The LV/BW value in patients with decreased counts at 48 weeks increased from 0.83 to 0.91 at 96 weeks after SVR. The median platelet count increased significantly from $8.0 \times 10^{4} / \mu \mathrm{L}$ at 48 weeks after SVR to $10.4 \times 10^{4} / \mu \mathrm{L}$ at 96 weeks after SVR $(P=0.020)$.

\section{Discussion}

In this study, we investigated the relationship between liver volume and the changes in platelet counts of Japanese patients with HCV infection treated with DAAs who obtained SVR. Following DAA therapy, not only spleen volume, but also liver volume was significantly decreased at SVR. Furthermore, the patients with decreased platelet counts at 48 weeks after SVR showed a greater reduction rate in liver volumes than patients with increased platelet counts. The reduction rate in liver volume was correlated with change in platelet count, while the reduction rate in spleen volume was correlated with change in albumin level. To the best of our knowledge, this is the first study to estimate changes in liver and spleen volumes by $\mathrm{CT}$, and investigate the relationships between liver volume and liver function in Japanese patients with HCV treated by DAAs after achievement of SVR.

In this study, the spleen volume was continuously reduced until 48 weeks after SVR. Spleen volume was reported to be related to the severity of fibrosis[16]. A previous study reported that platelet counts increased and spleen sizes decreased following IFN therapy[3]. Interventional management for splenomegaly by methods such as partial splenic artery embolization (PSE) or splenectomy leads to increased platelet counts and improved liver function[17]. In our study, the spleen volume decreased significantly, whether or not the platelet counts increased. However, change in spleen volume was significantly correlated with change in serum albumin level. Studies have shown that reduction in spleen volume by PSE reduced portal pressure and flow in the splenic vein, but maintained portal perfusion[1820]. These studies suggest that reduction in splenic arterial flow caused significant reduction in splenic venous flow with minimal decrease in the portal vein flow volume, while the flow of the superior mesenteric vein increased. The increased flow in the superior mesenteric vein led to improvement in liver function, including the level of serum albumin. We propose that the same mechanism underlies improvement in liver function after SVR achieved by DAA therapy.

Liver volume significantly decreased from baseline to SVR, and increased from 48 to 96 weeks after SVR in this study. The platelet counts correlated with the changes in liver volume and increased with liver volume. Chen et al reported that the volumes of the left, right, and caudate lobes tended to increase with exacerbation in inflammation and increased severity of fibrosis, but decreased in cirrhosis[11]. Another study reported that improvement in hepatic steatosis was accompanied by a significant decrease in the volumes of the left and right lobes, without significant change in spleen volume[21]. In our study, only 4 patients had fatty liver at baseline and the fatty liver of them were observed at 48 weeks after SVR too. For these reasons, the eradication of HCV by DAAs reduced hepatic inflammation, which might have led to reduction in liver volume. 
Platelets play an important role in liver regeneration. A previous study reported that patients with low platelet counts after hepatic resection showed decreased regeneration of liver compared with patients with normal or high platelet counts[22]. Several studies described the following effects due to platelets that resulted in regeneration of liver: direct effect on hepatocytes, where platelets translocate to the space of Disse and release growth factors through direct contact with hepatocytes; cooperation between platelets and sinusoidal endothelial cells, where the high concentration of sphingosine-1-phosphate in platelets induces excretion of interleukin- 6 from sinusoidal endothelial cells; and collaboration between platelets and Kupffer cells, where the functions of Kupffer cells are enhanced by platelets[23]. Plateletderived serotonin was recently reported to be relevant to liver regeneration[24]. The platelets recruited to sinusoidal endothelial cells or making cell-to-cell contact with hepatocytes release serotonin granules. It has been suggested that serotonin might act by an indirect liver regenerative effect, stimulating the release of a-granules from platelets[23-25].

The reduction in spleen volume also affects liver regeneration. Transforming growth factor (TGF)- $\beta$, which is derived from the spleen and hepatic stellate cells, was found to inhibit liver regeneration[26]. Asanoma et al have reported that TGF- $\beta 1$ and cytokines such as IL -6 , which are produced by macrophages in the spleen, might affect the progression of liver fibrosis and regeneration in patients with liver cirrhosis[17].

Decreased production of thrombopoietin (TPO) in the liver is another mechanism for thrombocytopenia. Cirrhotic patients with thrombocytopenia have lower levels of circulating TPO than patients without thrombocytopenia[27]. No published reports have mentioned an association between TPO and liver volume. However, since the liver is the main organ that produces TPO, a larger-volume liver might lead to higher levels of TPO.

In this study, the patients with decreased platelet count at 48 weeks after SVR showed greater reduction in liver volume than patients who increased that at SVR from baseline. The more inflammatory status, less reserved liver function or liver regenerating potential at baseline might affect the duration that reincrease the platelet counts and liver volume. Though it was not clarified in this study, portal hypertension may also affect these differences.

This study has limitations. This was a retrospective single-center study with insufficient numbers of participants in order to exclude the effect of antitumor therapy. Especially, the small sample size may over or underestimate the relationship between change in platelet count and change in LV/BW. The wide $95 \%$ confidential interval was also affected by small sample size. The measurement method of liver volume is also a limitation of this study. This study has risk of overestimate liver function because CT could not estimate how much of whole liver is function. We did not evaluate TPO or other liver regenerative factors at any time point. Additional studies that evaluate hematopoietic ability in relation to liver volume and platelet count are needed. Investigations are also needed to evaluate the association between liver volume and platelet count in patients with decompensated cirrhosis. And finally, further 
longitudinal studies are needed to investigate patient outcome including development HCC according to liver volumes or platelet counts.

In conclusion, the change in platelet count after SVR achieved by DAA therapy is related to the change in liver volume. Although most patients obtain increased platelet count and liver volume as a result of DAA therapy, patients showing slow improvement in platelet count might show reduction in the ability of the liver to regenerate. The platelet count after SVR obtained by DAA therapy should be considered to be a noninvasive liver marker of regeneration and should therefore be monitored over time.

\section{Declarations}

Ethics approval and consent to participate: This study was conducted in accordance with the 2013 Declaration of Helsinki and approved by the ethical committee of Kyoto Prefectural University of Medicine (reference number: ERB-C-588-1).

Consent for publication: Not applicable.

Availability of data and materials: The datasets used and/or analysed during the current study are available from the corresponding author on reasonable request.

Competing interests: The authors declare that they have no competing interests.

Funding: This study was not supported by any funding.

Authors' contributions: YS and YI contributed to the study design, data collection and analysis. YS was a major contributor in writing the manuscript. All authors contributed to data interpretation, drafting or revising the article, gave final approval of the version to be published, and agree to be accountable for all aspects of the work. All authors read and approved the final manuscript.

Acknowledgement: Not applicable

\section{References}

1. Wedemeyer H, Dore GJ, Ward JW. Estimates on HCV disease burden worldwide-filling the gaps. J Viral Hepat 2015;22(suppl 1):1-5

2. Ogawa E, Furusyo N, Nakamuta M et al. Glecaprevir and pibrentasvir for Japanese patients with chronic hepatitis $\mathrm{C}$ genotype 1 or 2 infection: Results from a multicenter, real-world cohort study. Hepatol Res 2019 Mar 8. doi: 10.1111/hepr.13328. [Epub ahead of print]

3. van derMeer AJ, Maan R, Veldt BJ et al. Improvement of platelets after SVR among patients with chronic HCV infection and advanced hepatic fibrosis. J Gastroenterol Hepatol 2016; 31: 1168-76.

4. Everson GT, Shiffman ML, Hoefs JC et al. Quantitative liver function tests improve the prediction of clinical outcomes in chronic hepatitis C: results from the Hepatitis C Antiviral Longterm Treatment 
against Cirrhosis Trial. Hepatology 2012; 55: 1019-1029.

5. Carr BI, Guerra V, Pancoska P. Thrombocytopenia in relation to tumor size in patients with hepatocellular carcinoma. Oncology 2012; 83: 339-345.

6. Pang Q, Qu K, Zhang JY et al. The prognostic value of platelet count in patients with hepatocellular carcinoma: a systematic review and meta-analysis. Medicine (Baltimore) 2015; 94:e1431.

7. Scheiner B, Kirstein M, Popp S et al. Association of Platelet Count and Mean Platelet Volume with Overall Survival in Patients with Cirrhosis and Unresectable Hepatocellular Carcinoma. Liver Cancer. 2019;8(3):203-217.

8. Chen TY, Chen CL, Huang TL et al. Remnant liver regeneration and spleen volume changes after living liver donation: influence of the middle hepatic vein. Clin Transplant 2006;20(6):725-31.

9. Cheng YF, Chen CL, Lai CY et al: Assessment of donor fatty livers for liver transplantation. Transplantation 2001 15;71(9):1221-5.

10. Zhou XP, Lu T, Wei YG et al. Liver volume variation in patients with virus-induced cirrhosis: findings on MDCT. Am J Roentgenol 2007;189:W153-W159.

11. Liu P, Li P, He W et al. Liver and spleen volume variations in patients with hepatic fibrosis. World J Gastroenterol 2009;15:3298-3302.

12. Hagan MT, Sayuk GS, Lisker-Melman M et al. Liver volume in the cirrhotic patient: does size matter? Dig Dis Sci 2014;59:886-891.

13. Ozaki K, Matsui O, Kobayashi S et al. Selective atrophy of the middle hepatic venous drainage area in hepatitis C-related cirrhotic liver: morphometric study by using multidetector CT. Radiology 2010;257:705-714.

14. Giannini E, Botta F, Borro P et al. Platelet count/spleen diameter ratio: proposal and validation of a non-invasive parameter to predict the presence of oesophageal varices in patients with liver cirrhosis. Gut 2003;52:1200-1205.

15. Berzigotti A, Seijo S, Arena U et al. Elastography, spleen size, and platelet count identify portal hypertension in patients with compensated cirrhosis. Gastroenterology 2013;144:102-111.

16. Tarao K, Hoshino H, Motohashi I et al. Changes in liver and spleen volume in alcoholic liver fibrosis of man. Hepatology 1989; 9: 589-593

17. Asanoma M, Ikemoto T, Mori H et al. Cytokine expression in spleen affects progression of liver cirrhosis through liverspleen cross-talk. Hepatol Res 2014; 44: 1217-23.

18. Helaly AZ, Al-Warraky MS, El-Azab GI et al. Portal and splanchnic hemodynamics after partial splenic embolization in cirrhotic patients with hypersplenism. APMIS 2015;123(12):1032-1039.

19. Nishida O, Moriyasu F, Nakamura T et al. Interrelationship between splenic and superior mesenteric venous circulation manifested by transient splenic arterial occlusion using a balloon catheter. Hepatology 1987; 7:442-6.

20. Mukaiya M, Hirata K, Yamashiro K et al. Changes in portal hemodynamics and hepatic function after partial splenic embolization (PSE) and percutaneous transhepatic obliteration (PTO). Cancer 
Chemother Pharmacol 1994;33(Suppl):S37-41.

21. Chen TY, Chen CL, Tsang LL et al. Correlation between hepatic steatosis, hepatic volume, and spleen volume in live liver donors. Transplant Proc 2008;40(8):2481-3.

22. Margonis GA, Amini N, Buettner S et al. Impact of early postoperative platelet count on volumetric liver gain and perioperative outcomes after major liver resection. Br J Surg 2016;103(7):899-907.

23. Meyer J, Lejmi E, Fontana $P$ et al. A focus on the role of platelets in liver regeneration: do plateletendothelial cell interactions initiate the regenerative process? J Hepatol 2015; 63:1263-1271.

24. Starlinger $P$, Assinger $A$, Haegele $S$ et al. Evidence for serotonin as a relevant inducer of liver regeneration after liver resection in humans. Hepatology 2014; 60: 257-266.

25. Lesurtel $\mathrm{M}$, Clavien PA. Platelet-derived serotonin: translational implications for liver regeneration. Hepatology 2014; 60: 30-33.

26. Nakamura T, Sakata $\mathrm{R}$, Ueno $\mathrm{T}$ et al. Inhibition of transforming growth factor $\beta$ prevents progression of liver fibrosis and enhances hepatocyte regeneration in dimethylnitrosamine-treated rats. Hepatology 32: 247-255, 2000.

27. Kaushansky K. Thrombopoietin. N Engl J Med 1998;339(11):746-54.

\section{Tables}

Table 1. Characteristics of 36 patients at start of DAA therapy. 


\begin{tabular}{|ll|}
\hline & $\mathrm{N}=36$ \\
\hline HCV genotype $(1 \mathrm{a} / 1 \mathrm{~b} / 2 \mathrm{a} / 2 \mathrm{~b}))$ & $1 / 33 / 1 / 1$ \\
\hline Gender (male/female)) & $23 / 13$ \\
\hline Age (years) & $68(48-85)$ \\
\hline BMI $\left(\mathrm{kg} / \mathrm{m}^{2}\right)$ & $21.7(16.4-30.1)$ \\
\hline Alcohol intake & $8(22.2 \%)$ \\
\hline Diabetes mellitus & $11(30.6 \%)$ \\
\hline Hypertension & $15(41.7 \%)$ \\
\hline Fatty liver & $4(11.1 \%)$ \\
\hline Esophageal varices & $9(25.0 \%)$ \\
\hline Past history of HCC & $22(61.1 \%)$ \\
\hline Total bilirubin, $\mathrm{mg} / \mathrm{dL}$ & $0.89(0.32-1.66)$ \\
\hline AST, U/L & $44(20-291)$ \\
\hline ALT, U/L & $35(10-175)$ \\
\hline GGT, g/dL & $28(12-132)$ \\
\hline Albumin, g/dL & $3.9(2.7-4.6)$ \\
\hline Platelet count, x 104/ $\mathrm{HL}$ & $10.3(4.5-29.9)$ \\
\hline Total cholesterol, $\mathrm{mg} / \mathrm{dL}$ & $154(115-231)$ \\
\hline Triglyceride, mg/dL & $82(46-204)$ \\
\hline LDL-C, mg/dL & $7.6(1.8-90.0)$ \\
\hline AFP, ng/mL & $5.08(1.50-32.52)$ \\
\hline FIB-4 index & $4.2(1.1-14.4)$ \\
\hline
\end{tabular}

Results are presented as n (\%) for qualitative data or as median (range) for quantitative data. 
Abbreviations: DAA, direct acting antivirals; BMI, body mass index; HCC, hepatocellular carcinoma; AST, aspartate aminotransferase; ALT, alanine aminotransferase; GGT, gamma-glutamyl transferase; LDL-; low-density lipoprotein cholesterol; AFP, alpha-fetoprotein

Table 2. Characteristics of 36 patients according to change in platelet count from baseline to after 48 weeks of SVR 


\begin{tabular}{|c|c|c|c|}
\hline & $\begin{array}{l}\text { Platelet count decreased } \\
\mathrm{n}=10\end{array}$ & $\begin{array}{l}\text { Platelet count increased } \\
n=26\end{array}$ & $\mathrm{p}$ \\
\hline Gender (male/female)) & $5 / 5$ & $18 / 8$ & 0.440 \\
\hline Age (years) & $72(59-77)$ & $70(48-85)$ & 0.876 \\
\hline $\mathrm{BMI}\left(\mathrm{kg} / \mathrm{m}^{2}\right)$ & $21.4(18.6-25.1)$ & $22.2(16.4-30.1)$ & 0.741 \\
\hline Alcohol intake & $1(10.0 \%)$ & $7(26.9 \%)$ & 0.397 \\
\hline Diabetes mellitus & $3(30.0 \%)$ & $8(30.8 \%)$ & 1.000 \\
\hline Hypertension & $4(40.0 \%)$ & $11(42.3 \%)$ & 1.000 \\
\hline Fatty liver & $1(10.0 \%)$ & $3(11.5 \%)$ & 1.000 \\
\hline Esophageal varices & $3(30.0 \%)$ & $6(23.1 \%)$ & 0.675 \\
\hline Past history of HCC & $5(50.0 \%)$ & $17(65.4 \%)$ & 0.462 \\
\hline \multicolumn{4}{|l|}{ At baseline } \\
\hline Total bilirubin, mg/dL & $1.02(0.61-1.66)$ & $0.85(0.32-1.26)$ & 0.080 \\
\hline AST, U/L & $41(21-77)$ & $42(20-291)$ & 0.337 \\
\hline ALT, U/L & $40(13-86)$ & $35(10-175)$ & 0.931 \\
\hline GGT, g/dL & $29(14-132)$ & $29(12-110)$ & 0.768 \\
\hline Albumin, g/dL & $3.9(3.3-4.6)$ & $4.0(2.7-4.6)$ & 0.876 \\
\hline Platelet count, $\times 10^{4} / \mu \mathrm{L}$ & $9.7(4.5-29.9)$ & $10.5(4.6-21.9)$ & 0.614 \\
\hline AFP, ng/mL & $8.2(2.4-90.0)$ & $5.7(1.8-55.9)$ & 0.377 \\
\hline FIB-4 index & $5.00(1.50-14.21)$ & $4.67(1.88-32.52)$ & 0.876 \\
\hline Liver volume/body weight, ml & $24.0(20.7-35.7)$ & $21.1(14.5-37.9)$ & 0.037 \\
\hline $\begin{array}{l}\text { Spleen volume/body weight, } \\
\mathrm{ml}\end{array}$ & $5.35(1.1-11.3)$ & 3.88 (1.1-14.4) & 0.433 \\
\hline $\begin{array}{l}\Delta \text { Liver volume/body weight, } \\
\%\end{array}$ & 87 (73-98) & $97(82-114)$ & $<0.001$ \\
\hline $\begin{array}{l}\triangle \text { Spleen volume/body } \\
\text { weight, } \%\end{array}$ & $89(58-117)$ & $85(53-144)$ & 0.543 \\
\hline
\end{tabular}


Results are presented as n (\%) for qualitative data or as median (range) for quantitative data. Abbreviations: SVR, sustained virological response; BMI, Body mass index; HCC, hepatocellular carcinoma; AST, aspartate aminotransferase; ALT, alanine aminotransferase; GGT, gamma-glutamyl transferase; AFP, alpha-fetoprotein.

Table 3. Factors associated with improvement in platelet count at 48 weeks after SVR from baseline evaluated by multivariate analysis

\begin{tabular}{|c|c|c|c|}
\hline & \multirow[t]{2}{*}{ Category } & \multicolumn{2}{|c|}{ Multivariate analysis } \\
\hline & & OR $(95 \% \mathrm{Cl})^{a}$ & $P$ value \\
\hline \multirow[t]{2}{*}{ Platelet count } & $1:<10 \times 10^{4} / \mu \mathrm{L}$ & & \multirow[t]{2}{*}{0.122} \\
\hline & $2: \geq 10 \times 10^{4} / \mu \mathrm{L}$ & & \\
\hline \multirow[t]{2}{*}{ Albumin } & $1:<3.8 \mathrm{~g} / \mathrm{dL}$ & & \multirow[t]{2}{*}{0.413} \\
\hline & $2: \geq 3.8 \mathrm{~g} / \mathrm{dL}$ & & \\
\hline \multirow[t]{2}{*}{ Total bilirubin } & $1: \geq 1.0 \mathrm{mg} / \mathrm{dL}$ & & \multirow[t]{2}{*}{0.225} \\
\hline & $2:<1.0 \mathrm{mg} / \mathrm{dL}$ & & \\
\hline \multirow[t]{2}{*}{$\Delta$ Liver volume/body weight } & $1:<95 \%$ & 1 & \multirow[t]{2}{*}{0.005} \\
\hline & $2: \geq 95 \%$ & $76.9(3.78-1000)$ & \\
\hline
\end{tabular}

Abbreviations: OR, odds ratio; $\mathrm{Cl}$, confidence interval

\section{Figures}


Figure 1

AST

a.

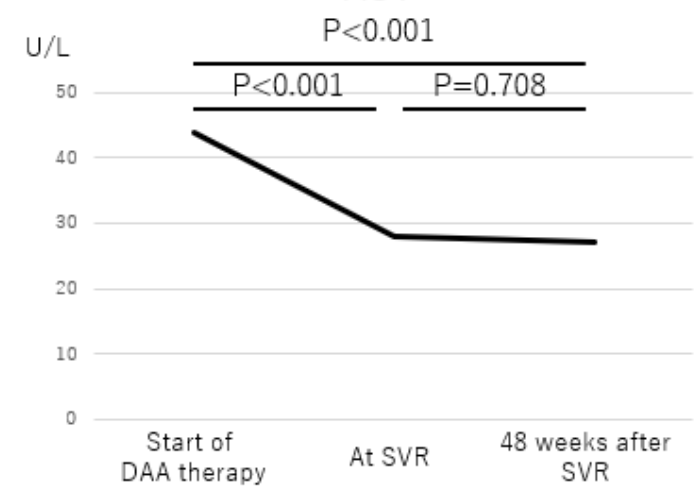

C.

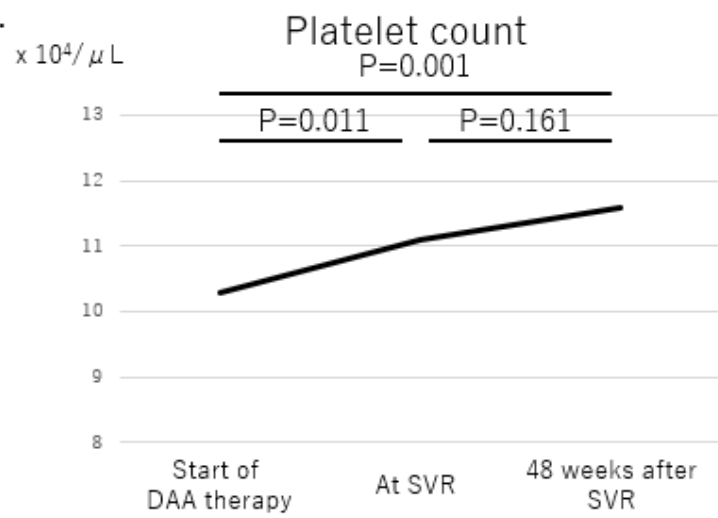

ALT

b.



d.



\section{Figure 1}

The clinical parameters of patients treated by direct-acting antiviral (a. AST, b. ALT, c. Platelet Counts, d. Albumin) at start of treatment, sustained virological response, and 48 weeks after SVR. 
Figure 2

a.

Liver volume/body weight

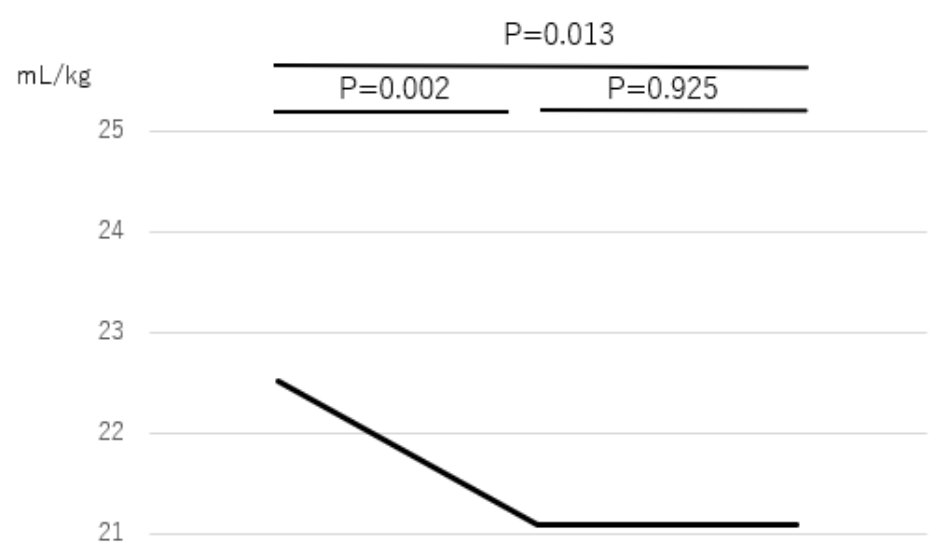

20

At SVR

b. Spleen volume/body weight

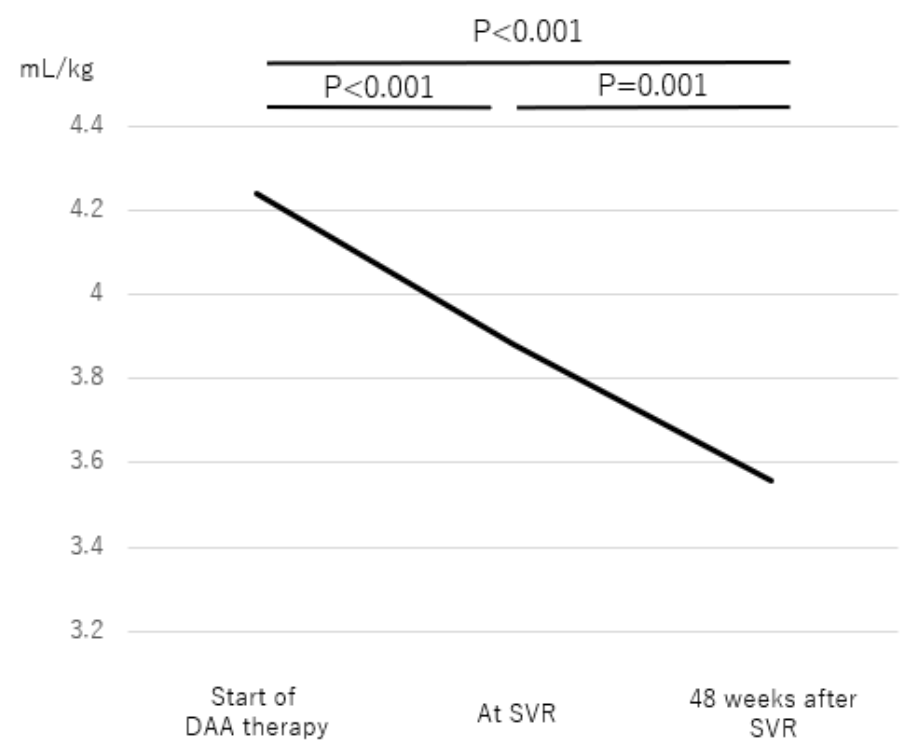

\section{Figure 2}

The clinical parameters of patients treated by direct-acting antiviral (a. liver volume/body weight, $b$. spleen volume/body weight) at start of treatment, sustained virological response (SVR), and 48 weeks after SVR 
Figure 3

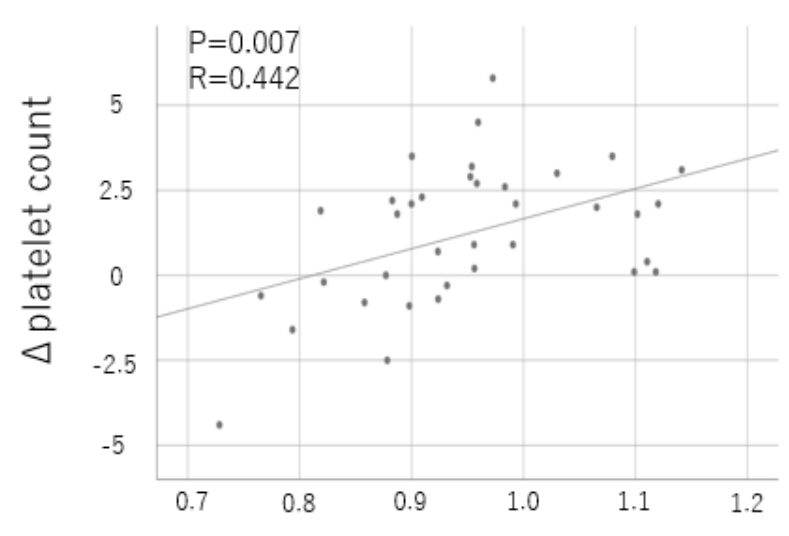

\%Liver volume

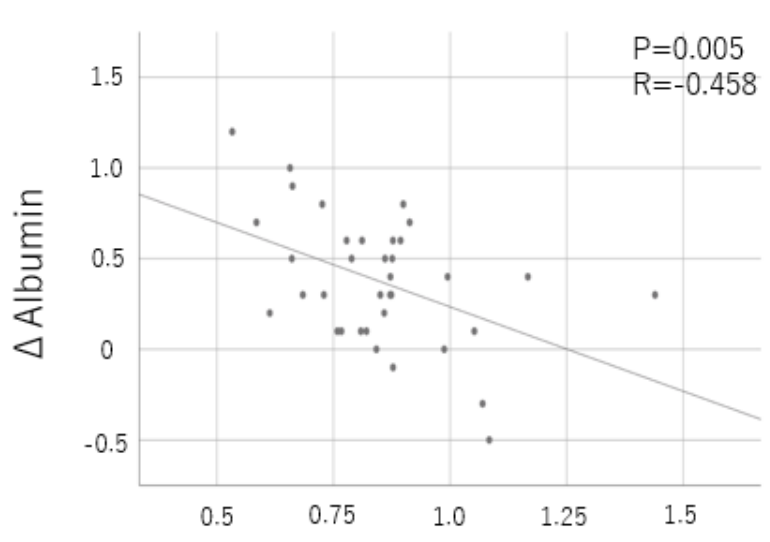

\%Spleen volume

\section{Figure 3}

Association between a. change in liver volume and platelet count, b. change in spleen volume and albumin level.

Figure 4

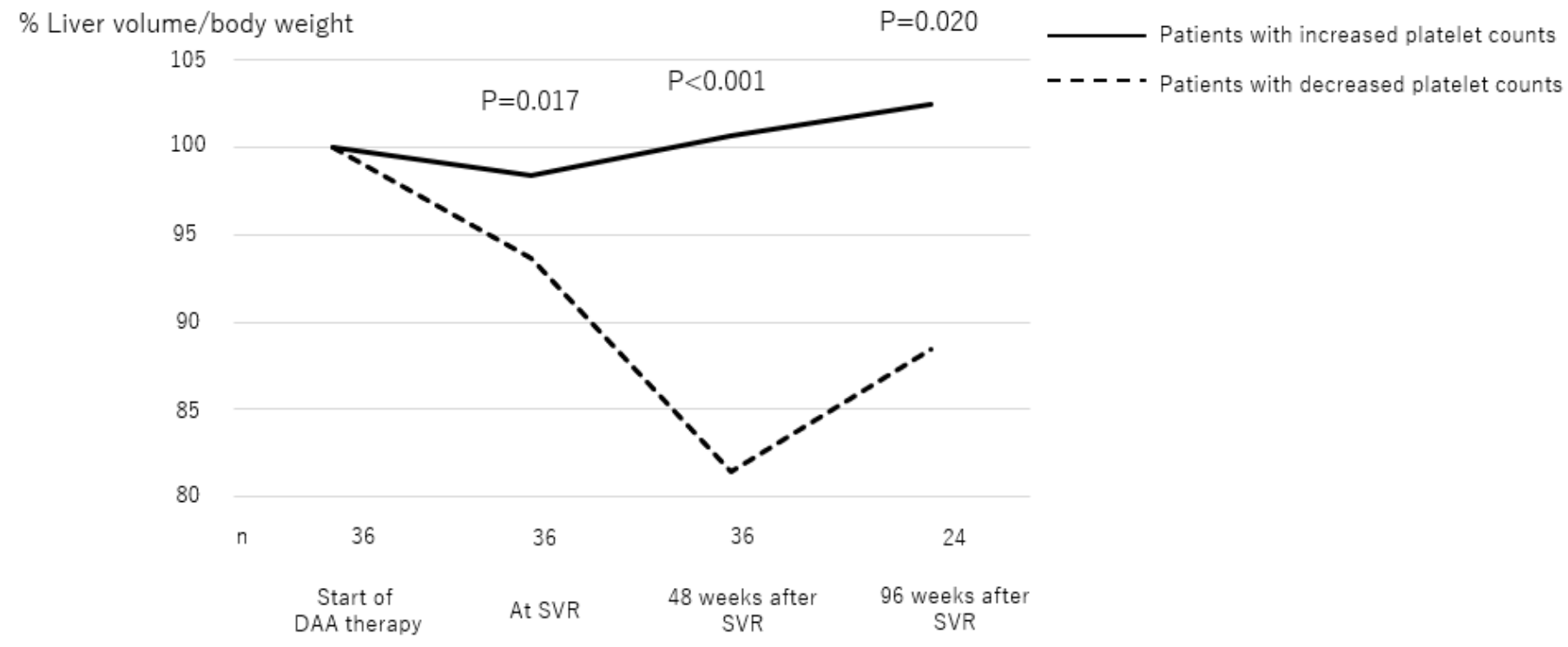




\section{Figure 4}

The percent liver volume/body weight according to change in platelet counts from baseline to 48 weeks after sustained virological response from baseline. 